\title{
NECESSARY CONDITIONS FOR LOCAL AND GLOBAL EXISTENCE TO A REACTION-DIFFUSION SYSTEM WITH FRACTIONAL DERIVATIVES
}

\author{
KAMEL HAOUAM AND MOURAD SFAXI
}

Received 6 August 2006; Revised 21 September 2006; Accepted 10 October 2006

We give some necessary conditions for local and global existence of a solution to reactiondiffusion system of type (FDS) with temporal and spacial fractional derivatives. As in the case of single equation of type (STFE) studied by M. Kirane et al. (2005), we prove that these conditions depend on the behavior of initial conditions for large $|x|$.

Copyright (c) 2006 Hindawi Publishing Corporation. All rights reserved.

\section{Introduction}

This paper deals with the following reaction-diffusion system of type (FDS):

$$
\text { (FDS) } \begin{cases}\mathbf{D}_{0 \mid t}^{\alpha} u+(-\Delta)^{\beta / 2} u=|v|^{p} & \text { in } \mathbb{R}^{N} \times \mathbb{R}^{+}, \\ \mathbf{D}_{0 \mid t}^{\delta} \nu+(-\Delta)^{\gamma / 2} v=|u|^{q} & \text { in } \mathbb{R}^{N} \times \mathbb{R}^{+}\end{cases}
$$

where $N \geq 1, p$ and $q$ are two positive reals.

For $\alpha \in(0,1)$ (resp., $\delta \in(0,1)$ ), " $\mathbf{D}_{0 \mid t}^{\alpha}$ " (resp., " $\mathbf{D}_{0 \mid t}^{\delta}$ ") denotes the time derivative of order $\alpha$ (resp., $\delta$ ) in the sense of Caputo (see Definition 1.2, see also [9]). While, for $\beta \in$ $[1,2]$ (resp., $\gamma \in[1,2])$, “ $(-\Delta)^{\beta / 2}$ ” (resp., “ $(-\Delta)^{\gamma / 2}$ ”) stands for the $\beta / 2$-fractional (resp., $\gamma / 2$-fractional) power of the Laplacian with respect to $x$ and defined by

$$
(-\Delta)^{\beta / 2} v(x)=\mathscr{F}^{-1}\left(|\xi|^{\beta} \mathscr{F}(v)(\xi)\right)(x),
$$

where $\mathscr{F}_{\mathscr{F}}$ denotes the Fourier transform and $\mathscr{F}^{-1}$ its inverse.

The system (FDS) is completed by the following initial conditions:

$$
u(\cdot, 0)=u_{0}, \quad v(\cdot, 0)=v_{0},
$$

and we will assume that both $u_{0}$ and $v_{0}$ are nonnegative continuous functions.

The system (FDS) was considered in the case $\alpha=\delta=1, \beta=\gamma=2$, by many authors in several contexts, see $[2,3,5]$ (with $\nu=\mu=1$ ). Moreover, concerning nonexistence result 
and basing one's argument on [4], Escobedo and Herero proved in [2] that if $p q \geq 1$, then the only global solution of the system (FDS), reduced to the following reaction-diffusion problem:

$$
\begin{aligned}
& u_{t}-\Delta u=v^{p}, \\
& v_{t}-\Delta v=u^{q},
\end{aligned}
$$

is the trivial one, that is, $u \equiv v \equiv 0$, while, in a recent paper (see [7]), the authors study the system (FDS) and they find a bound on $N$ leading to the absence of global nonnegative solutions. More precisely, recovering the case studied in [2] (when $\alpha=\delta=1, \beta=\gamma=2$ ), they proved the following.

THEOREM 1.1. If $p>1$ and $q>1$ and supposing

$$
N \leq \max \left\{\frac{\delta / q+\alpha-(1-1 / p q)}{\delta / \gamma q p^{\prime}+\alpha / \beta q^{\prime}}, \frac{\alpha / p+\delta-(1-1 / p q)}{\alpha / \beta p q^{\prime}+\delta / \gamma p^{\prime}}\right\},
$$

then the system (FDS) does not admit nontrivial global weak nonnegative solution.

Therefore, they also establish some necessary conditions for the existence of local and global solutions to the following problem:

$$
\text { (STFE) } \begin{cases}\mathbf{D}_{0 \mid t}^{\alpha} u+(-\Delta)^{\beta / 2} u=h|u|^{p} & \text { in } \mathbb{R}^{N} \times \mathbb{R}^{+}, \\ u(\cdot, 0)=u_{0} \geq 0 & \text { in } \mathbb{R}^{N},\end{cases}
$$

and these conditions depend on the behavior of the initial data $u_{0}$ and on the function $h$ for large $|x|$. Similar results can be found in [6] and [1].

Our results can be viewed as analogous to those obtained in [7], since the system (FDS) can be considered as a system of two equations of type (STFE). Therefore, we can extend these results to the more general system,

$$
\begin{gathered}
\mathbf{D}_{0 \mid t}^{\alpha} u+(-\Delta)^{\beta / 2}\left(|u|^{m-1} u\right)=h|v|^{p}+g|u|^{r} \quad \text { in } \mathbb{R}^{N} \times \mathbb{R}^{+}, \\
\mathbf{D}_{0 \mid t}^{\delta} v+(-\Delta)^{\gamma / 2}\left(|v|^{m-1} v\right)=k|u|^{q}+l|v|^{s} \quad \text { in } \mathbb{R}^{N} \times \mathbb{R}^{+}
\end{gathered}
$$

under some suitable conditions on $h, g, k$, and $l$. Of course, taking such form for reaction terms, all results presented here will depend also on the functions $h, g, k$, and $l$.

For the convenience of the reader, we recall here some definitions and properties of fractional derivatives in the sense of Caputo and of Riemann-Liouville.

Definition 1.2. The left-handed derivative and the right-handed derivative in the sense of Caputo for $\psi^{\prime} \in L^{1}(0, T)$ are defined, respectively, by

$$
\begin{gathered}
\left(\mathbf{D}_{0 \mid t}^{\alpha} \psi\right)(t)=\frac{1}{\Gamma(1-\alpha)} \int_{0}^{t} \frac{\psi^{\prime}(t)}{(t-\sigma)^{\alpha}} d \sigma, \\
\left(\mathbf{D}_{t \mid T}^{\alpha} \psi\right)(t)=-\frac{1}{\Gamma(1-\alpha)} \int_{t}^{T} \frac{\psi^{\prime}(t)}{(\sigma-t)^{\alpha}} d \sigma,
\end{gathered}
$$

where $\Gamma$ denotes, as usual, the Euler gamma function. 
Up to replace $\psi^{\prime}$ by $\psi$ and to keep the derivative operator before the integral in expressions (1.8), we obtain the definitions of the left-handed derivative and the right-handed derivative in the sense of Riemann-Liouville denoted, respectively, by $D_{0 \mid t}^{\alpha}$ and $D_{t \mid T}^{\alpha}$. See [8] for more details.

Recall that the Caputo derivative is related to the Riemann-Liouville one by the following formula:

$$
\mathbf{D}_{0 \mid t}^{\alpha} \psi(t)=D_{0 \mid t}^{\alpha}\{\psi(t)-\psi(0)\}
$$

Finally, taking into account the following integration by parts formula:

$$
\int_{0}^{T}\left(D_{0 \mid t}^{\alpha} f\right)(t) g(t) d t=\int_{0}^{T} f(t)\left(D_{t \mid T}^{\alpha} g\right)(t) d t
$$

we adopt the following.

Definition 1.3. For $0<T \leq \infty$, it is said that $(u, v)$ is a local weak solution to (FDS) defined on $Q_{T}\left(Q_{T}:=\mathbb{R}^{N} \times(0, T)\right)$ if

$$
\begin{aligned}
& u \in C\left([0, T] ; L_{\mathrm{loc}}^{1}\left(\mathbb{R}^{N}\right)\right) \cap L^{q}\left(Q_{T}, d x d t\right), \\
& v \in C\left([0, T] ; L_{\mathrm{loc}}^{1}\left(\mathbb{R}^{N}\right)\right) \cap L^{p}\left(Q_{T}, d x d t\right),
\end{aligned}
$$

and satisfies

$$
\begin{gathered}
\int_{Q_{T}}|v|^{p} \varphi+\int_{Q_{T}} u_{0} D_{t \mid T}^{\alpha} \varphi=\int_{Q_{T}} u D_{t \mid T}^{\alpha} \varphi+\int_{Q_{T}} u(-\Delta)^{\beta / 2} \varphi \\
\int_{Q_{T}}|u|^{q} \psi+\int_{Q_{T}} v_{0} D_{t \mid T}^{\delta} \psi=\int_{Q_{T}} v D_{t \mid T}^{\delta} \psi+\int_{Q_{T}} v(-\Delta)^{\gamma / 2} \psi
\end{gathered}
$$

for all test functions $\varphi, \psi \in C_{x, t}^{2,1}\left(Q_{T}\right)$ satisfying $\varphi(\cdot, T)=\psi(\cdot, T)=0$. If $T=+\infty$, it is said that $(u, v)$ is a global weak solution.

\section{Statement of the results}

Our main result is the following.

Theorem 2.1. Assume that $p, q>1$ and let $(u, v)$ be a local solution $(T<+\infty)$ of problem (FDS). Then, the following estimates hold:

$$
\begin{aligned}
& \liminf _{|x| \rightarrow \infty} u_{0}(x) \leq C T^{-(\alpha+p \delta) /(p q-1)}, \\
& \liminf _{|x| \rightarrow \infty} v_{0}(x) \leq C^{\prime} T^{-(\delta+q \alpha) /(p q-1)},
\end{aligned}
$$

where $C$ and $C^{\prime}$ are some positive constants. 
4 Necessary conditions for local and global existence

Proof. Thanks to variational formulation (1.12), we have

$$
\begin{gathered}
\int_{Q_{T}} u_{0} D_{t \mid T}^{\alpha} \varphi \leq \int_{Q_{T}} u D_{t \mid T}^{\alpha} \varphi+\int_{Q_{T}} u(-\Delta)^{\beta / 2} \varphi \\
\int_{Q_{T}} v_{0} D_{t \mid T}^{\delta} \psi \leq \int_{Q_{T}} v D_{t \mid T}^{\delta} \psi+\int_{Q_{T}} v(-\Delta)^{\gamma / 2} \psi
\end{gathered}
$$

for all nonnegative test functions $\varphi, \psi \in C_{x, t}^{2,1}\left(Q_{T}\right)$ satisfying $\varphi(\cdot, T)=\psi(\cdot, T)=0$.

Using Hölder's inequality, we get

$$
\begin{gathered}
\int_{Q_{T}} u\left|D_{t \mid T}^{\alpha} \varphi\right| \leq\left(\int_{Q_{T}}|u|^{q} \psi\right)^{1 / q}\left(\int_{Q_{T}}\left|D_{t \mid T}^{\alpha} \varphi\right|^{q^{\prime}} \psi^{-q^{\prime} / q}\right)^{1 / q^{\prime}}, \\
\int_{Q_{T}} u\left|(-\Delta)^{\beta / 2} \varphi\right| \leq\left(\int_{Q_{T}}|u|^{q} \psi\right)^{1 / q}\left(\int_{Q_{T}}\left|(-\Delta)^{\beta / 2} \varphi\right|^{q^{\prime}} \psi^{-q^{\prime} / q}\right)^{1 / q^{\prime}} .
\end{gathered}
$$

And thus

$$
\int_{Q_{T}} u_{0} D_{t \mid T}^{\alpha} \varphi \leq\left(\int_{Q_{T}}|u|^{q} \psi\right)^{1 / q} \mathscr{A}
$$

where

$$
\mathscr{A}=\left(\int_{Q_{T}}\left|D_{t \mid T}^{\alpha} \varphi\right|^{q^{\prime}} \psi^{-q^{\prime} / q}\right)^{1 / q^{\prime}}+\left(\int_{Q_{T}}\left|(-\Delta)^{\beta / 2} \varphi\right|^{q^{\prime}} \psi^{-q^{\prime} / q}\right)^{1 / q^{\prime}} .
$$

As above, using once again Hölder's inequality, we obtain

$$
\int_{Q_{T}} v_{0} D_{t \mid T}^{\delta} \psi \leq\left(\int_{Q_{T}}|v|^{p} \varphi\right)^{1 / p} \mathscr{B}
$$

where

$$
\mathscr{B}=\left(\int_{Q_{T}}\left|D_{t \mid T}^{\delta} \psi\right|^{p^{\prime}} \varphi^{-p^{\prime} / p}\right)^{1 / p^{\prime}}+\left(\int_{Q_{T}}\left|(-\Delta)^{\gamma / 2} \psi\right|^{p^{\prime}} \varphi^{-p^{\prime} / p}\right)^{1 / p^{\prime}} .
$$

Furthermore, keeping the first terms in the left-hand sides of (1.12) and recalling that both $u_{0}$ and $v_{0}$ are nonnegative functions, we obtain as above

$$
\begin{gathered}
\int_{Q_{T}}|v|^{p} \varphi \leq\left(\int_{Q_{T}}|u|^{q} \psi\right)^{1 / q} \mathscr{A}, \\
\int_{Q_{T}}|u|^{q} \psi \leq\left(\int_{Q_{T}}|v|^{p} \varphi\right)^{1 / p} \mathscr{B} .
\end{gathered}
$$


Consequently,

$$
\begin{aligned}
& \left(\int_{Q_{T}}|v|^{p} \varphi\right)^{1-1 / p q} \leq \mathscr{B}^{1 / q} \mathscr{A}, \\
& \left(\int_{Q_{T}}|u|^{q} \psi\right)^{1-1 / p q} \leq \mathscr{B} \mathscr{A}^{1 / p} .
\end{aligned}
$$

Applying (2.10) and (2.11), respectively, in (2.5) and (2.7), we get

$$
\begin{aligned}
& \left(\int_{Q_{T}} u_{0} D_{t \mid T}^{\alpha} \varphi\right)^{1-1 / p q} \leq \mathscr{B}^{1 / q} \mathscr{A}, \\
& \left(\int_{Q_{T}} v_{0} D_{t \mid T}^{\delta} \psi\right)^{1-1 / p q} \leq \mathscr{B} \mathscr{A}^{1 / p} .
\end{aligned}
$$

Now, we consider some test functions in (2.12) and (2.13), introduced in [7], of the form

$$
\varphi(x, t)=\psi(x, t)=\Phi\left(\frac{x}{R}\right) \begin{cases}\left(1-\frac{t}{T}\right)^{l}, & 0<t \leq T, \\ 0, & t>T,\end{cases}
$$

where $\Phi \in W^{1, \infty}\left(\mathbb{R}^{N}\right)$ is nonnegative, with support in $\{R<|x|<2 R\}$ and satisfies

$$
\begin{array}{ll}
\left((-\Delta)^{\beta / 2} \Phi\right)_{+} \leq k \Phi & \text { for some constant } k>0 \\
\left((-\Delta)^{\gamma / 2} \Phi\right)_{+} \leq h \Phi & \text { for some constant } h>0
\end{array}
$$

The exponent $l$, introduced in (2.14), is any positive real number if

$$
\min \left(p-\frac{1}{1-\delta}, q-\frac{1}{1-\alpha}\right) \geq 0
$$

and $l>\max \left(\alpha q^{\prime}-1, \delta p^{\prime}-1\right)$ if

$$
\min \left(p-\frac{1}{1-\delta}, q-\frac{1}{1-\alpha}\right)<0
$$

where $p^{\prime}$ and $q^{\prime}$ are, respectively, the conjugate exponents of $p$ and $q$.

Moreover, note that

$$
D_{t \mid T}^{\alpha} \varphi(x, t)=\Lambda T^{-\alpha} \Phi\left(\frac{x}{R}\right)\left(1-\frac{t}{T}\right)^{l-\alpha},
$$

where $\Lambda:=\Gamma(1+l) / \Gamma(1+l-\alpha)$.

Similarly,

$$
D_{t \mid T}^{\delta} \psi(x, t)=\Upsilon T^{-\delta} \Phi\left(\frac{x}{R}\right)\left(1-\frac{t}{T}\right)^{l-\delta},
$$

where $\Upsilon:=\Gamma(1+l) / \Gamma(1+l-\delta)$. 
Necessary conditions for local and global existence

Next, as in [7], consider the change of variables

$$
t=T \tau, \quad x=R y .
$$

We get

$$
\int_{Q_{T}} u_{0} D_{t \mid T}^{\alpha} \varphi d x d t=\frac{\Lambda T^{1-\alpha} R^{N}}{l-\alpha+1} \int_{\mathbb{R}^{N}} u_{0}(R y) \Phi(y) d y .
$$

Taking into account (2.15), we obtain

$$
\mathscr{A} \leq\left(\frac{\Lambda^{q^{\prime}} T^{1-\alpha q^{\prime}} R^{N}}{(l-\alpha) q^{\prime}-l\left(q^{\prime} / q\right)+1} \int_{\mathbb{R}^{N}} \Phi(y) d y\right)^{1 / q^{\prime}}+\left(\frac{T R^{-\beta q^{\prime}+N} k^{q^{\prime}}}{l+1} \int_{\mathbb{R}^{N}} \Phi(y) d y\right)^{1 / q^{\prime}}
$$

or

$$
\mathscr{A} \leq R^{N / q^{\prime}}\left\{\frac{\Lambda T^{1 / q^{\prime}-\alpha}}{\left[(l-\alpha) q^{\prime}-l\left(q^{\prime} / q\right)+1\right]^{1 / q^{\prime}}}+\frac{T^{1 / q^{\prime}} R^{-\beta} k}{(l+1)^{1 / q^{\prime}}}\right\}\left(\int_{\mathbb{R}^{N}} \Phi(y) d y\right)^{1 / q^{\prime}} .
$$

Analogously, using once again (2.15), we get

$$
\mathscr{B} \leq R^{N / p^{\prime}}\left\{\frac{\Upsilon T^{1 / p^{\prime}-\delta}}{\left[(l-\delta) p^{\prime}-l\left(p^{\prime} / p\right)+1\right]^{1 / p^{\prime}}}+\frac{T^{1 / p^{\prime}} R^{-\gamma} h}{(l+1)^{1 / p^{\prime}}}\right\}\left(\int_{\mathbb{R}^{N}} \Phi(y) d y\right)^{1 / p^{\prime}} .
$$

Hence, (2.21), (2.23), and (2.24) with inequality (2.12) lead to

$$
\begin{aligned}
& T^{(1-\alpha)(1-1 / p q)}\left\{\int_{\mathbb{R}^{N}} u_{0}(R y) \Phi(y) d y\right\}^{1-1 / p q} \\
& \quad \leq\left(C_{1} T^{1 / q^{\prime}-\alpha}+C_{2} T^{1 / q^{\prime}} R^{-\beta}\right)\left(C_{3} T^{1 / p^{\prime}-\delta}+C_{4} T^{1 / p^{\prime}} R^{-\gamma}\right)^{1 / q}\left(\int_{\mathbb{R}^{N}} \Phi(y) d y\right)^{1-1 / p q},
\end{aligned}
$$

where $C_{1}, C_{2}, C_{3}$, and $C_{4}$ are positive constants independent on $R$ and $T$. Consequently,

$$
T^{(1-\alpha)(1-1 / p q)}\left\{\inf _{|y|>1} u_{0}(R y)\right\}^{1-1 / p q} \leq\left(C_{1} T^{1 / q^{\prime}-\alpha}+C_{2} T^{1 / q^{\prime}} R^{-\beta}\right)\left(C_{3} T^{1 / p^{\prime}-\delta}+C_{4} T^{1 / p^{\prime}} R^{-\gamma}\right)^{1 / q} .
$$

Finally, letting $R \rightarrow+\infty$ in (2.26), we conclude that

$$
T^{(1-\alpha)(1-1 / p q)}\left\{\liminf _{|x| \rightarrow \infty} u_{0}(x)\right\}^{1-1 / p q} \leq C T^{1 / q^{\prime}-\alpha} T^{1 / p^{\prime} q-\delta / q}
$$

Estimate (2.1) is then proved.

Using estimate (2.13) and applying the change of variables $t=T \tau$ and $x=R y$ in the expressions of $\mathscr{A}$ and $\mathscr{B}$, we obtain estimate (2.2). 
In the sequel, we also assume that $p, q>1$.

Concerning existence of global and local solution, we give the following necessary condition results.

COROLLARY 2.2. Suppose that the system (FDS) admits a nontrivial global nonnegative weak solution. Then

$$
\liminf _{|x| \rightarrow \infty} u_{0}(x)=\liminf _{|x| \rightarrow \infty} v_{0}(x)=0
$$

Corollary 2.3. If $\liminf _{|x| \rightarrow \infty} u_{0}(x)=+\infty$ or if $\liminf _{|x| \rightarrow \infty} v_{0}(x)=+\infty$, then the system (FDS) cannot have nontrivial local nonnegative weak solution.

Corollary 2.4. If $A:=\liminf _{|x| \rightarrow \infty} u_{0}(x)>0$ and $B:=\liminf _{|x| \rightarrow \infty} v_{0}(x)>0$, then

$$
T^{(\alpha+p \delta) /(p q-1)} \leq \frac{C}{A}, \quad T^{(\delta+p \alpha) /(p q-1)} \leq \frac{C^{\prime}}{B},
$$

where $C$ and $C^{\prime}$ denote the constants introduced in (2.1) and (2.2).

Our second main result is the following.

THEOREM 2.5. Supposing that problem (FDS) admits a nontrivial global nonnegative weak solution, then there exist positive constants $H$ and $K$ such that

$$
\begin{aligned}
& \liminf _{|x| \rightarrow \infty} u_{0}(x)|x|^{(\alpha+p \delta) /(p q-1)} \leq H, \\
& \liminf _{|x| \rightarrow \infty} v_{0}(x)|x|^{(\delta+q \alpha) /(p q-1)} \leq K .
\end{aligned}
$$

Proof. Let us return to expression (2.25) and multiply by $|x|^{(\alpha+p \delta) /(p q-1)}|x|^{-(\alpha+p \delta) /(p q-1)}$ inside the integral of both members. Taking into account the fact that $\operatorname{supp} \Phi \subset\{1<|y|<$ $2\}$, expression (2.25) becomes

$$
\begin{aligned}
& T^{(1-\alpha)(1-1 / p q)} \inf _{|x|>R}\left\{u_{0}(x)|x|^{(\alpha+p \delta) /(p q-1)}\right\}^{1-1 / p q} \\
& \quad \leq\left(C_{1} T^{1 / q^{\prime}-\alpha}+C_{2} T^{1 / q^{\prime}} R^{-\beta}\right)\left(C_{3} T^{1 / p^{\prime}-\delta}+C_{4} T^{1 / p^{\prime}} R^{-\gamma}\right)^{1 / q}(2 R)^{(\alpha+p \delta) / p q} .
\end{aligned}
$$

Taking $T=R$, it follows

$$
\inf _{|x|>R}\left\{u_{0}(x)|x|^{(\alpha+p \delta) /(p q-1)}\right\}^{1-1 / p q} \leq 2^{(\alpha+p \delta) / p q}\left(C_{1}+C_{2} R^{\alpha-\beta}\right)\left(C_{3}+C_{4} R^{\delta-\gamma}\right)^{1 / q} .
$$

Since $\alpha<\beta$ and $\delta<\gamma$, we pass to the limit with respect to $R$ and we obtain

$$
\left\{\liminf _{|x| \rightarrow \infty} u_{0}(x)|x|^{(\alpha+p \delta) /(p q-1)}\right\}^{1-1 / p q} \leq 2^{(\alpha+p \delta) / p q} C_{1} C_{3}^{1 / q} .
$$

Hence, inequality (2.30) holds for $H:=2^{(\alpha+p \delta) /(p q-1)}\left\{C_{1} C_{3}^{1 / q}\right\} p q /(p q-1)$. As before, we obtain inequality $(2.31)$ with $K:=2^{(\delta+q \alpha) /(p q-1)}\left\{C_{1}^{1 / p} C_{3}\right\}^{p q /(p q-1)}$. 


\section{References}

[1] P. Baras and R. Kersner, Local and global solvability of a class of semilinear parabolic equations, Journal of Differential Equations 68 (1987), no. 2, 238-252.

[2] M. Escobedo and M. A. Herrero, Boundedness and blow up for a semilinear reaction-diffusion system, Journal of Differential Equations 89 (1991), no. 1, 176-202.

[3] M. Fila, H. A. Levine, and Y. Uda, A Fujita-type global existence-global non-existence theorem for a system of reaction diffusion equations with differing diffusivities, Mathematical Methods in the Applied Sciences 17 (1994), no. 10, 807-835.

[4] H. Fujita, On the blowing up of solutions of the Cauchy problem for $u_{t}=\Delta u+u^{1+\alpha}$, Journal of the Faculty of Science. University of Tokyo. Section IA 13 (1966), 109-124 (1966).

[5] V. A. Galaktionov, S. P. Kurdyumov, and A. A. Samarskiĭ, A parabolic system of quasilinear equations. I, Differentsial'nye Uravneniya 19 (1983), no. 12, 2123-2140 (Russian).

[6] A. S. Kalashnikov, The heat equation in a medium with nonuniformly distributed nonlinear heat sources or absorbers, Vestnik Moskovskogo Universiteta. Seriya I. Matematika, Mekhanika (1983), no. 3, 20-24.

[7] M. Kirane, Y. Laskri, and N.-E. Tatar, Critical exponents of Fujita type for certain evolution equations and systems with spatio-temporal fractional derivatives, Journal of Mathematical Analysis and Applications 312 (2005), no. 2, 488-501.

[8] J. D. Munkhammar, Riemann-Liouville fractional derivatives and the Taylor-Riemann series, Project Report, Department of Mathematics, Uppsala University, Uppsala, 2004.

[9] I. Podlubny, Fractional Differential Equations, Mathematics in Science and Engineering, vol. 198, Academic Press, California, 1999.

Kamel Haouam: Latp, Centre de Mathématiques et Informatiques, Université de Provence, 39 rue F., 13453 Marseille cedex 13, France

E-mail address: haouam@cmi.univ-mrs.fr

Mourad Sfaxi: Latp, Centre de Mathématiques et Informatiques, Université de Provence, 39 rue F., 13453 Marseille cedex 13, France

E-mail address: sfaxi@cmi.univ-mrs.fr 


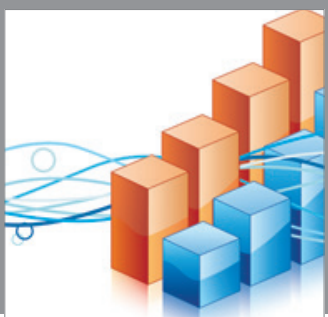

Advances in

Operations Research

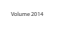

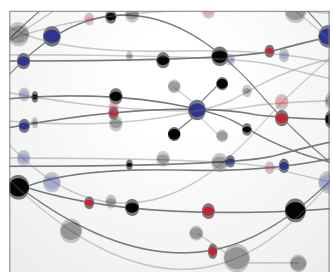

\section{The Scientific} World Journal
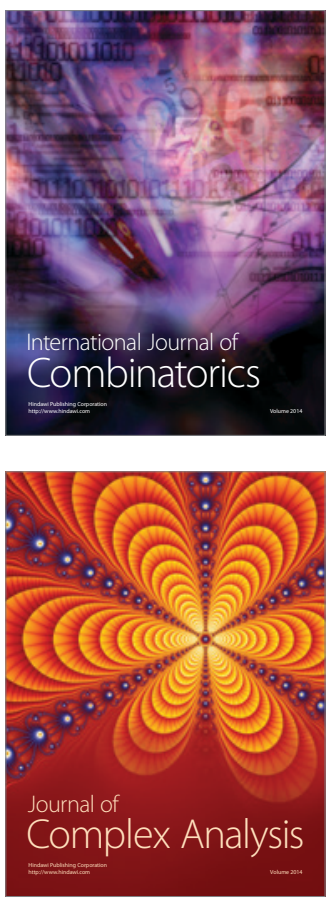

International Journal of

Mathematics and

Mathematical

Sciences
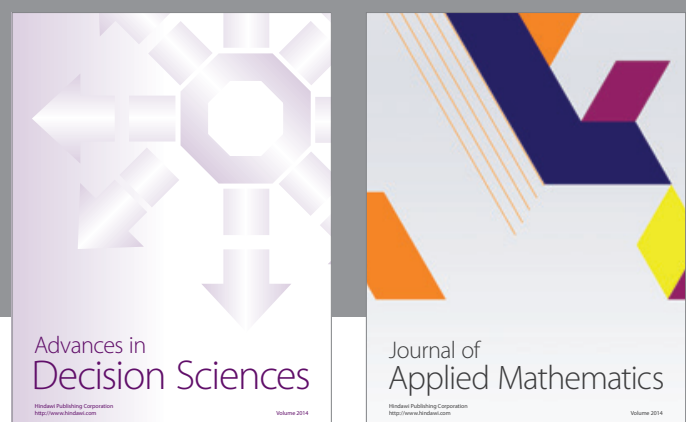

Journal of

Applied Mathematics
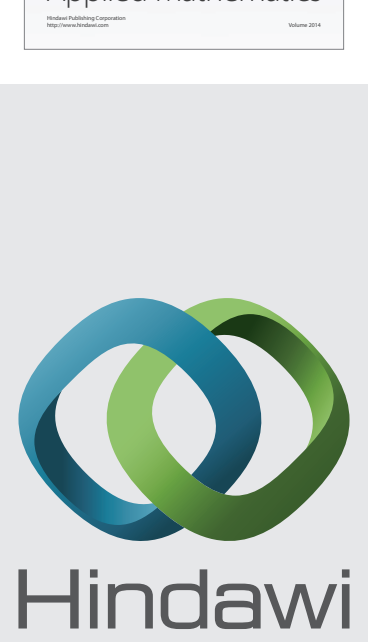

Submit your manuscripts at http://www.hindawi.com
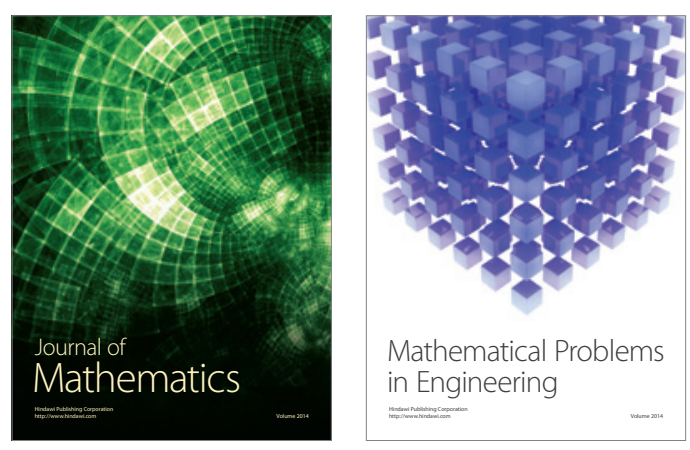

Mathematical Problems in Engineering
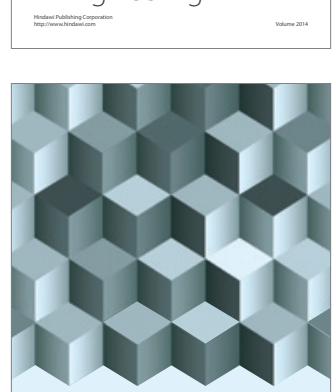

Journal of

Function Spaces
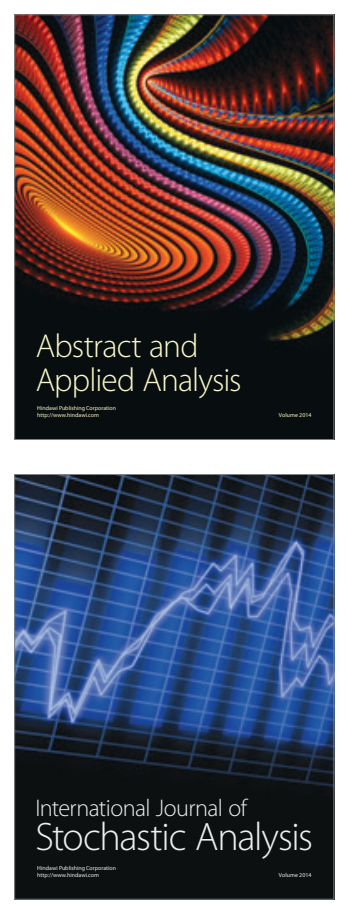

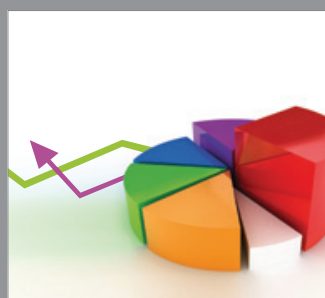

ournal of

Probability and Statistics

Promensencen
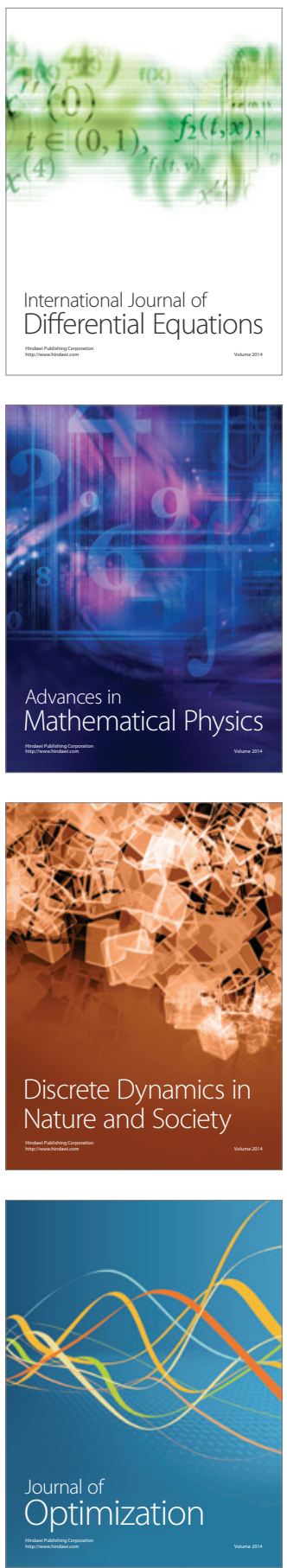